\title{
Editorial
}

\section{5-methylcytosine - The fifth base of DNA: The fifth wheel on a car or a highly promising diagnostic and therapeutic target in cancer?}

\author{
Martin Widschwendter* \\ Department of Gynaecological Oncology, Institute for Women's Health, University College London, EGA Hospital, \\ 2nd Floor Huntley Street, London WC1E 6DH, UK
}

Keywords: DNA methylation, epigenetics, 5-methylcytosine, biomarkers

The concept of early detection of tumours before they spread and become incurable, represents one of the most important challenges in reducing the impact of the growing burden of cancer worldwide. Whilst improvements in the methods of treating cancer have had some success, it is clear that tumour stage at diagnosis has a major effect on survival for all types of cancer. A non-invasive marker for cancer that will achieve early diagnosis as well as predicting prognosis and response to treatment and providing insight into aetiology, would be a powerful clinical tool. Recently, this search has intensified due to the introduction of novel molecular technologies with the potential to identify cellular changes at the epigenome level.

In the late 1970s, Peter A. Jones and coworkers were the first to discover the role of DNA methylation in cellular differentiation. Over the last 25 years, epigenetics, a term covering hypomethylation, hypermethylation, loss of imprinting and chromatin modification, has been recognised to be as important as genetics in human cancer.

This Special Issue of Disease Markers, appears at a critical time in the development of the field of cancer

*Corresponding author. Tel.: +44 207380 6807; Fax: +44 20 7380 9748; E-mail: M.Widschwendter@ucl.ac.uk. epigenetics and can serve as an important bridge between the converging fields of cancer biomarkers and epigenetics (Fig. 1). The issue contains 10 articles covering: epidemiological epigenetics; DNA methylation changes of specific genes and cancer types; applications of epigenetic markers (which will be introduced in the clinics in the near future); and an excellent overview of biostatistical challenges associated with the arrival of these new markers.

A huge variety of different epigenetic alterations have been documented in lung, prostate, ovarian and colorectal cancer (reviewed by Keith M. Kerr, Janice S. Galler, Jeffrey A. Hagen, Peter W. Laird and Ite A. Laird-Offringa; Vera L. Costa, Rui Henrique and Carmen Jerónimo; Gordon Strathdee; and Marion Zitt, Matthias Zitt and Hannes M. Müller; respectively). Several genes (e.g. RASSF1A, reviewed by Luke B. Hesson, Wendy N. Cooper and Farida Latif) demonstrate abnormal methylation in a broad spectrum of tumours, which highlights the importance of switching off certain pathways during carcinogenesis.

The potential of DNA-methylation-based technologies to contribute to clinical diagnostics and indirectly to therapeutics as predictors of response to therapy is reviewed by Heidi Fiegl and Karim Elmasry. There are several important advantages for DNA methylation based markers: 


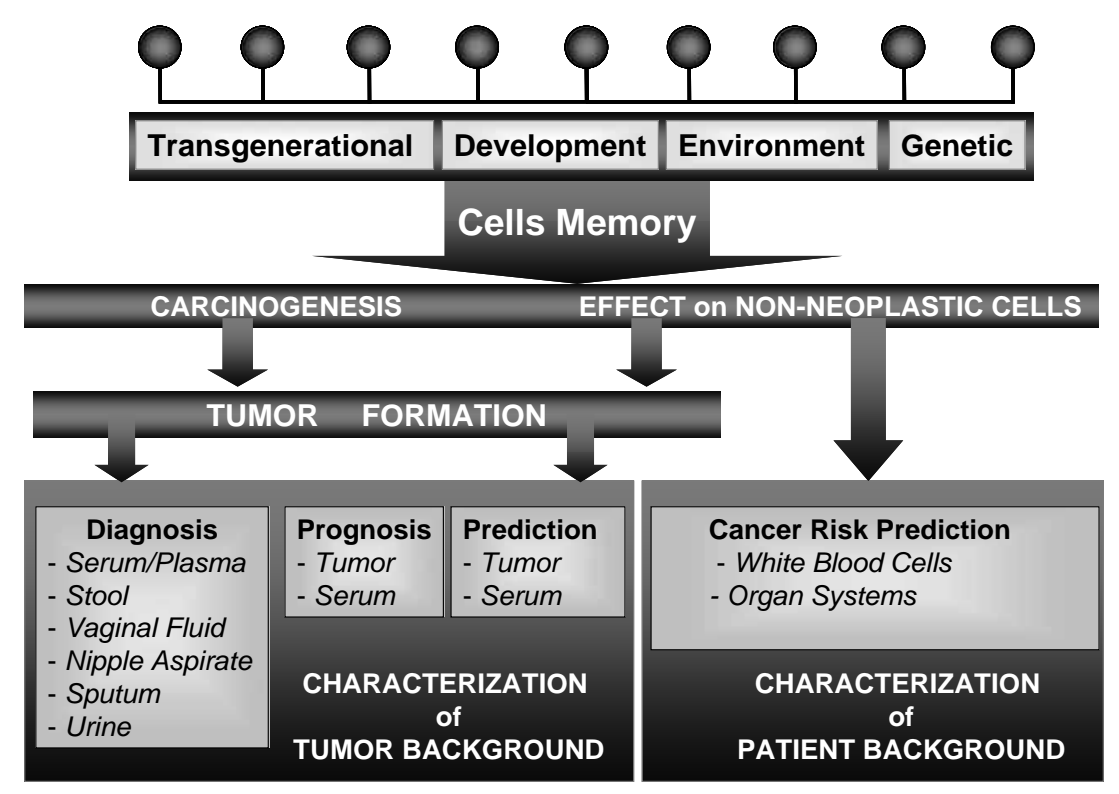

Fig. 1. Epigenetics: Causes, effects and potential clinical applications in cancer.

- Compared to RNA- or protein-based markers, DNA represents a stable molecule that can be isolated and amplified from various sources (WBCs, vaginal secretion, serum, sputum, nipple aspirate, stool, urine, paraffin embedded tissue).

- DNA methylation may act as a surrogate marker, integrating and in the long-term, reflecting genetic as well as non-genetic causes (transgenerational, developmental and environmental influences) that eventually lead to cancer.

- Abnormal DNA methylation is a chemically and biologically stable molecule that can be readily detected, acting as a surrogate marker for gene expression.

- CpG islands (CpG islands are approximately 1$\mathrm{kb}$ stretches of DNA-containing clusters of $\mathrm{CpG}$ dinucleotides that are usually unmethylated in normal cells and become methylated in cancer) are frequently confined to small specific regions within genes (usually the promoter region) and are not "randomly" spread throughout the gene.

- The availability of 25-30,000 CpG islands allows the development of a variety of markers that can simultaneously be analysed on the same platform.

- Recently invented technology allows DNA methylation markers to be analyzed on a high throughput platform.

All these features have led to development of new analyses, some of which are already being tested in clinical trials. Epigenetic silencing of the MGMT gene encoding a DNA repair enzyme was recently found to be of predictive value in a randomised clinical trial for newly diagnosed glioblastoma testing the addition of the alkylating agent temozolomide to standard radiotherapy (reviewed by Peter Hau, Roger Stupp and Monika E. Hegi). Proof of principle studies to detect early cancer via DNA methylation analysis in serum, stool, vaginal fluid, nipple aspirate, sputum and urine have been performed and large ongoing validation studies are on the way.

One of the most interesting questions to emerge as a consequence of initial epigenetic studies is whether epigenetic changes analysed in normal tissue can reflect an individuals risk of developing cancer. Recently, in a proof of principle study, loss of imprinting of IGF2 in peripheral blood cells was associated with increased risk of developing human colorectal cancer (reviewed by Hengmi Cui).

The wealth of information gained by DNA methylation analysis has become a challenging task for biostatisticians and Todd A. Alonzo and Kimberly D. Siegmund provide an excellent review about various statistical approaches.

A sharp increase in the identification of novel DNA methylation markers contrasts greatly with our lack of knowledge about the causes for epigenetic alterations. The recent emergence of "epigenetic epidemiology" aims to explain these influences and intends to use them to direct epigenetics toward improved health and longevity (reviewed by Craig A. Cooney). 
These 10 chapters therefore capture several of the most exciting recent developments in this rapidly evolving field. The addition of methyl groups to our DNA, and the changes that occur as we age and develop cancer, provides us with important new therapeutic strategies. Altered patterns of DNA methylation can be detected with high sensitivity, potentially providing us with prognostic information, and can be reversed by appropriate drug treatment. These possibilities make cancer epigenetics a most exciting field of current translational research. 


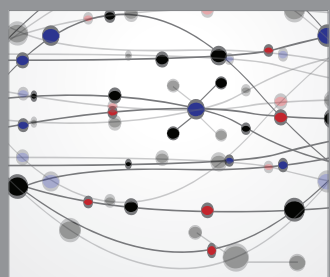

The Scientific World Journal
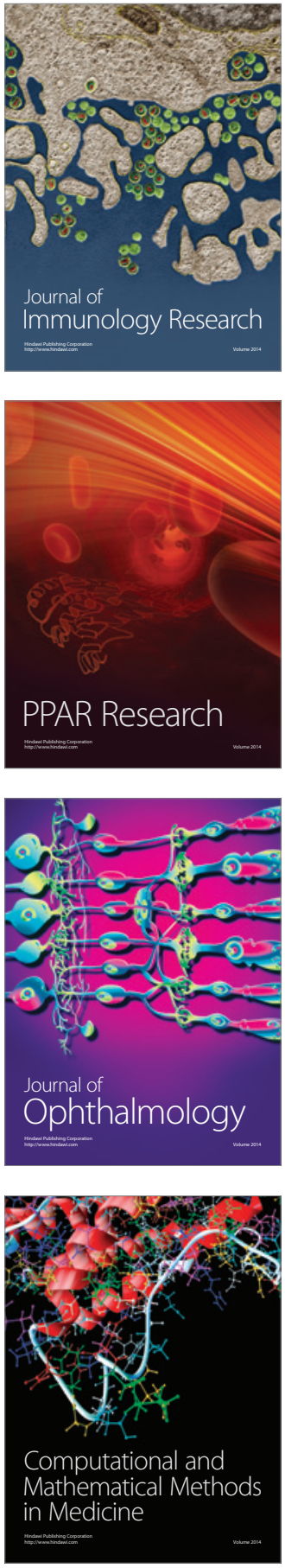

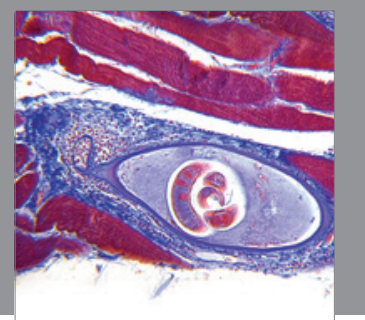

Gastroenterology

Research and Practice
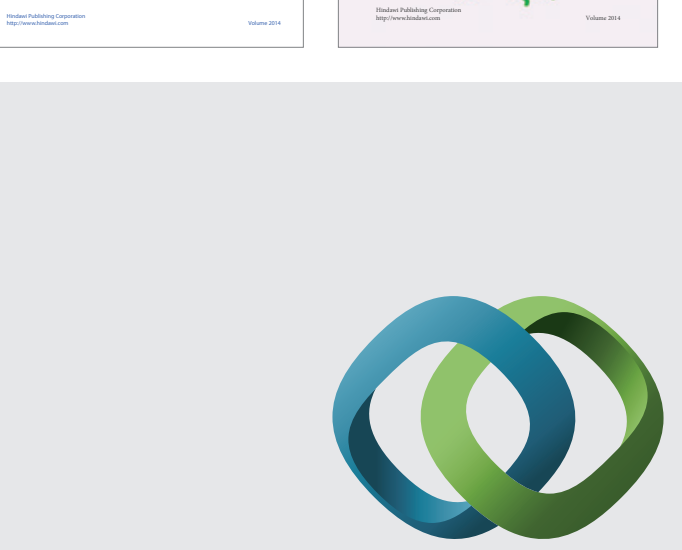

\section{Hindawi}

Submit your manuscripts at

http://www.hindawi.com
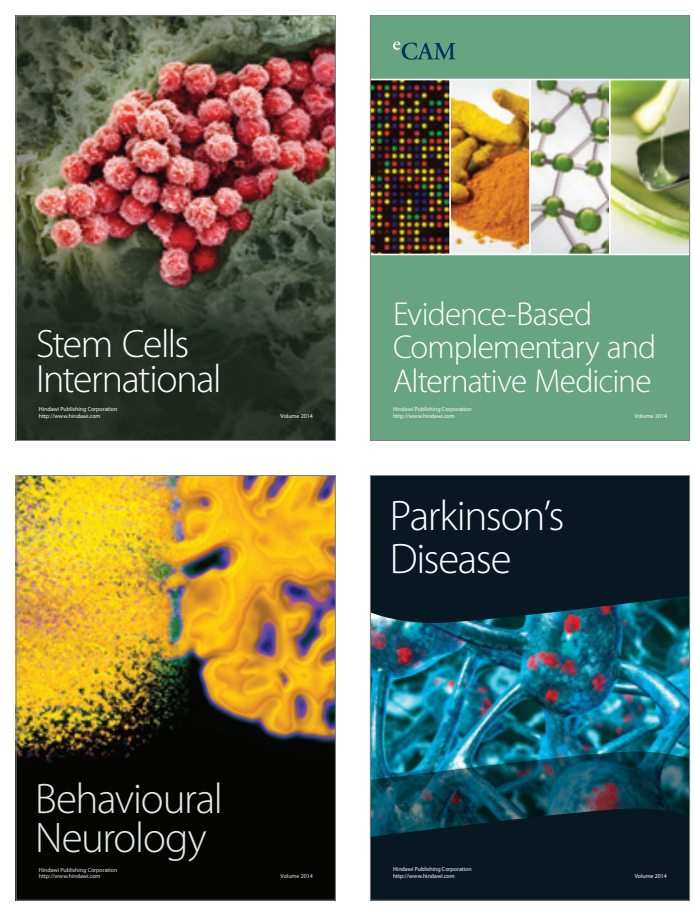

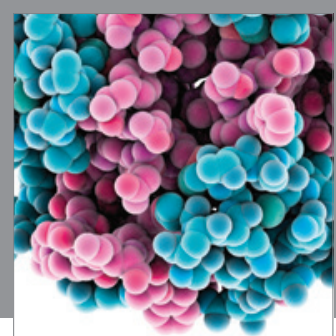

Journal of
Diabetes Research

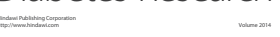

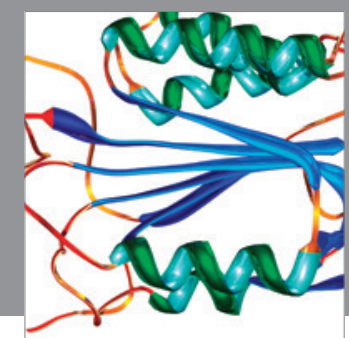

Disease Markers
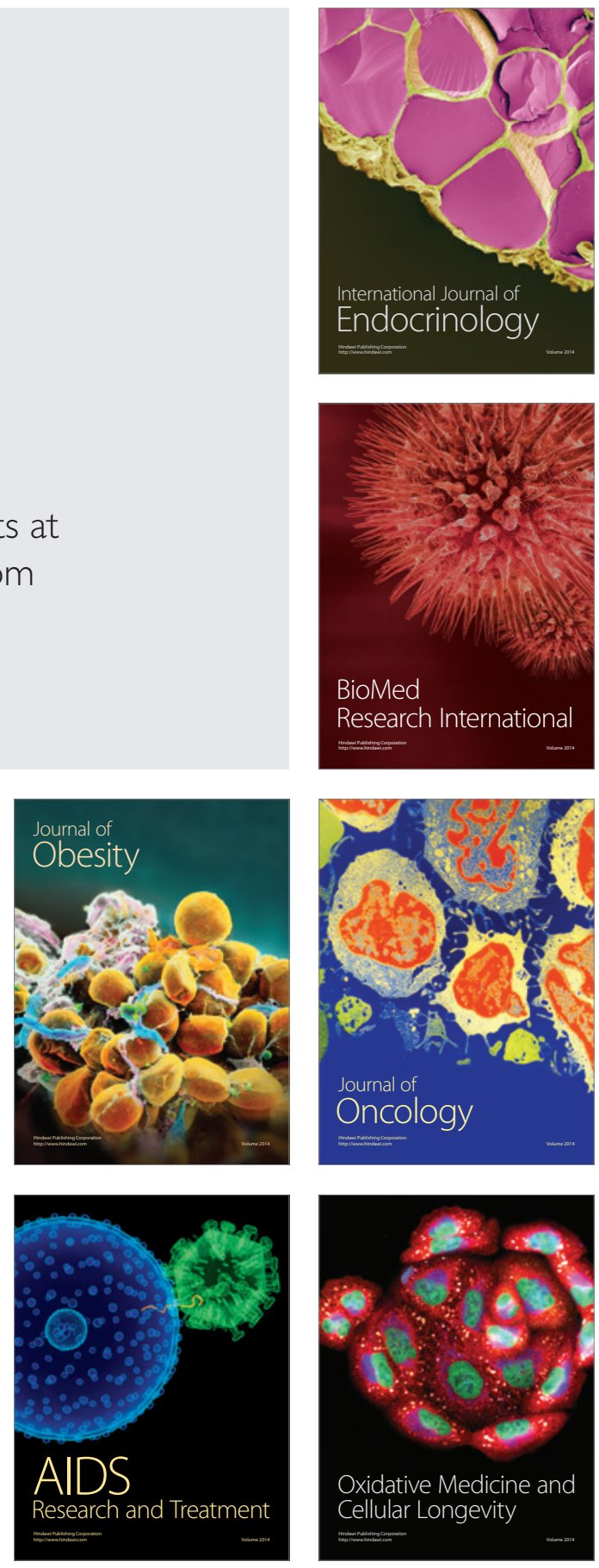